\title{
Small Vessel Transient Ischemic Attack and Lacunar Infarction Detected with Perfusion-Weighted MRI
}

\author{
Hyun-Wook Nah \\ Cerebrovascular Center and Department of Neurology, Dong-A University College of Medicine, Busan, Korea
}

\section{Dear Sir:}

The clinical diagnosis of transient ischemic attack (TIA) or minor stroke is highly subjective, especially if the brain imaging does not reveal any evidence of ischemic lesion. Herein, I report a case of small vessel TIA and another case of lacunar infarction in which the ischemic lesions were detected with the perfusion-weighted magnetic resonance imaging (MRI).

A 76-year-old woman presented with transient right side weakness. She experienced slurred speech and weakness of her right arm and leg for 10 minutes. On neurological examination, she did not show any deficit. The initial multimodal MRI, taken 4 hours after the symptom, revealed no abnormality on diffusion-weighted imaging (DWI) but showed perfusion defect in her left corona radiata and basal ganglia. The intracranial and extracranial magnetic resonance angiography (MRA) did not show significant vascular lesion related to the perfusion defect. The patient did not experience further symptom. However, the follow-up DWI 2 days later revealed diffusion restriction corresponding to the initial perfusion defect (Figure 1A-F).

A 66-year-old hypertensive woman presented with the clinical findings of cheiro-oral syndrome. Neurological examination revealed sensory deficit in her left fingers and left hemi-mouth. MRI taken 12 hours after the symptom onset revealed no abnormality on DWI but showed perfusion defect in her right lateral thalamus. MRA did not show any vascular lesion related to the perfusion defect. Her symptom persisted and the follow-up DWI 2 days later showed acute lacunar infarction in the right thalamus, which corresponded to the initial perfusion defect (Figure 1G-L).

The identification of ischemic lesion is important among patients with suspected TIA or minor stroke., ${ }^{1,2}$ This report shows
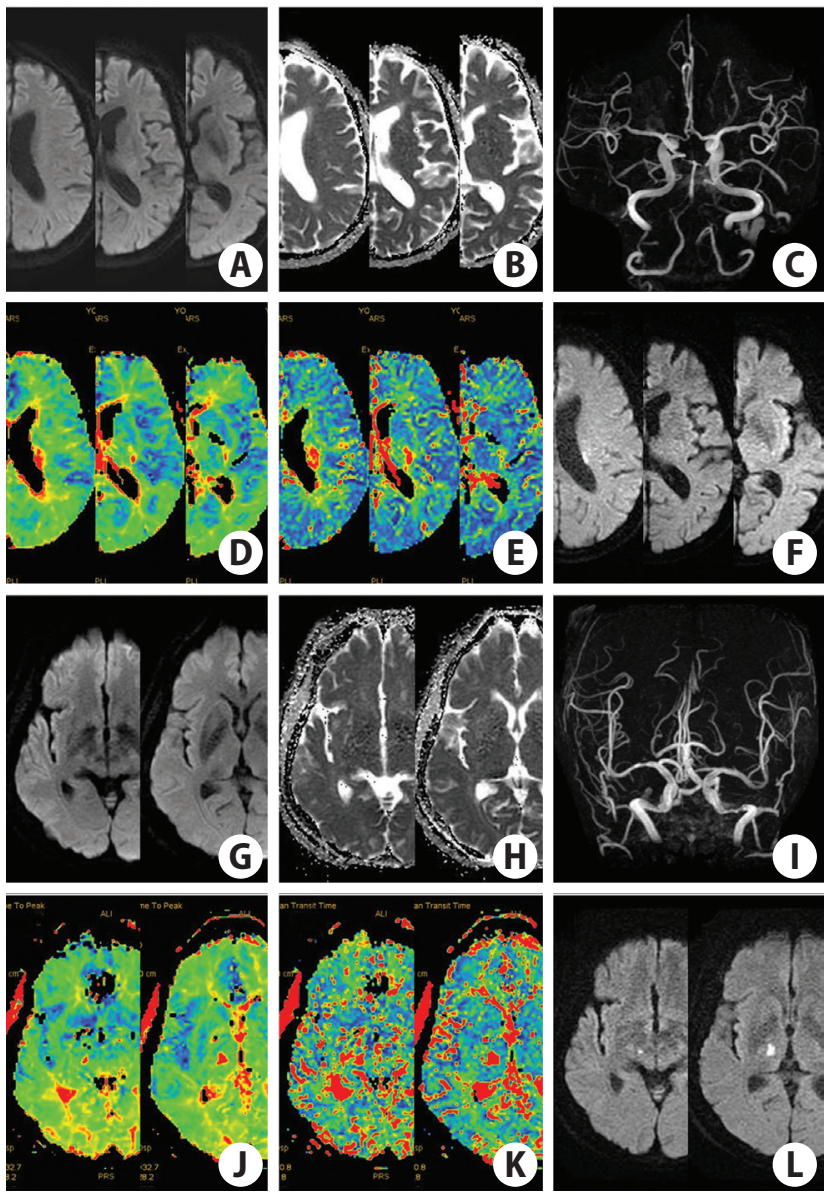

Figure 1. Multimodal MRI of patient with TIA (A-F) and cheiro-oral syndrome (G-L). The initial MRI of patient with TIA revealed no significant abnormality on diffusion-weighted imaging (DWI) (A), ADC map (B), and MR angiography (C) but showed perfusion defect in left corona radiata and basal ganglia on timeto-peak (D) and mean-transit-time map (E). The DWI obtained 2 days later revealed diffusion lesion in the left corona radiata and tail of left basal ganglia (F). The initial MRI of patient with cheiro-oral syndrome revealed no abnormality on DWI (G), ADC map (H), and MR angiography (I) but showed time delay in right thalamus on time-to-peak $(\mathrm{J})$ and mean-transit-time map (K). The DWI obtained 2 days later revealed diffusion restriction in right thalamus (L). MRI, magnetic resonance imaging; TIA, transient ischemic attack; $A D C$, apparent diffusion coefficients; MR, magnetic resonance. 
the usefulness of perfusion-weighted imaging (PWI) in detecting the ischemic lesions caused probably by small vessel diseases, which presented clinically as TIA and lacunar syndrome. It is reported that up to one third of TIA patients have a DWI lesion. ${ }^{3}$ The time from symptom onset to imaging could be a factor related with initial DWI positivity in TIA or minor stroke. Our previous study showed that time from symptom to DWI was shorter in DWI negative than DWI positive TIA patients, ${ }^{4}$ and another study also showed low DWI detection rate in the early ( 1 to 12 hours) phase after TIA compared with the later timeframe. ${ }^{5}$ The pathophysiology of delayed DWI lesions in our cases could be explained by the concept of penumbra in small vessel occlusive diseases. ${ }^{6}$ The penumbra which was shown initially as perfusion defect may have turned to true ischemic lesion. Whereas PWI has been known to be helpful for detecting ischemic lesions caused by large vessel disease and cardioembolism in DWI negative patients, ${ }_{1}^{4,7}$ our cases show its usefulness for small vessel occlusive diseases as well.

\section{References}

1. Uehara T, Minematsu K. Guidelines for management of patients with transient ischemic attack. Front Neurol Neurosci 2014;33:103-114.

2. Kim BJ, Kang HG, Kim HJ, Ahn SH, Kim NY, Warach S, et al. Magnetic resonance imaging in acute ischemic stroke treatment. J Stroke 2014;16:131-145.

3. Easton JD, Saver JL, Albers GW, Alberts MJ, Chaturvedi S,

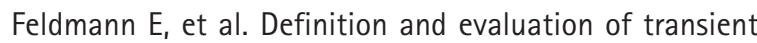
ischemic attack: a scientific statement for healthcare professionals from the American Heart Association/American Stroke Association Stroke Council; Council on Cardiovascular
Surgery and Anesthesia; Council on Cardiovascular Radiology and Intervention; Council on Cardiovascular Nursing; and the Interdisciplinary Council on Peripheral Vascular Disease. Stroke 2009;40:2276-2293.

4. Nah HW, Kwon SU, Kang DW, Lee DH, Kim JS. Diagnostic and prognostic value of multimodal MRI in transient ischemic attack. Int J Stroke 2014;9:895-901.

5. Uno $H$, Nagatsuka K, Kokubo Y, Higashi M, Yamada N, Umesaki $A$, et al. Detectability of ischemic lesions on diffusionweighted imaging is biphasic after transient ischemic attack. J Stroke Cerebrovasc Dis 2015;24:1059-1064.

6. Duering $M$, Csanadi $E$, Gesierich $B$, Jouvent $E$, Hervé $D$, Seiler $S$, et al. Incident lacunes preferentially localize to the edge of white matter hyperintensities: insights into the pathophysiology of cerebral small vessel disease. Brain 2013;136:27172726.

7. Zaharchuk G, Olivot JM, Fischbein NJ, Bammer R, Straka M, Kleinman JT, et al. Arterial spin labeling imaging findings in transient ischemic attack patients: comparison with diffusion- and bolus perfusion-weighted imaging. Cerebrovasc Dis 2012;34:221-228.

Correspondence: Hyun-Wook Nah

Department of Neurology, Dong-A University College of Medicine, 26 Daeshingongwon-ro, Seo-gu, Busan 49201, Korea

Tel: +82-51-240-2615

Fax: +82-51-244-8338

E-mail: hyunwook.nah@gmail.com

Received: June 22, 2016

Revised: August 14, 2016

Accepted: August 15, 2016

The authors have no financial conflicts of interest. 